\title{
Explotación y precariedad sociolaboral, la realidad de las personas migrantes trabajadoras en agricultura en España
}

\author{
Exploitation and socio-labor insecurity, the reality of \\ migrant agricultural workers in Spain
}

Erica Briones-Vozmediano ${ }^{1,2,3}$ (1) 0000-0001-8437-2781

Agustín González-González ${ }^{4,5}$ (i) 0000-0002-2189-6518

'Departamento y Facultad de Enfermería y Fisioterapia, Universidad de Lleida, Lleida, España.

${ }^{2}$ Grupo de estudios en Sociedad, Educación, Calidad de Vida y Salud (GESEC), Universidad de Lleida, Lleida, España.

${ }^{3}$ Grupo de investigación en cuidados en salud (GRECS), Instituto de Investigación Biomédica (IRB) de Lleida., Lleida, España.

${ }^{4}$ Departamento de Pedagogía, Universidad de Lleida, Lleida, España.

${ }^{5}$ Grupo de investigación y análisis social y educativo (GR-ASE), Universidad de Lleida, Lleida, España.

Fechas · Dates

Recibido: 01/12/2021

Aceptado: 03/01/2022

Publicado: 17/01/2022
Correspondencia · Corresponding Author

Erica Briones Vozmediano

E-mail: erica.briones.voz@gmail.com 
El trabajo agrícola de temporada en España se caracteriza por un gran desgaste físico, un bajo requerimiento de cualificación y unas precarias condiciones laborales. Estos elementos han generado que los autóctonos hayan ido abandonando paulatinamente este nicho laboral para ser remplazados por migrantes internacionales de una amplia variedad de orígenes ${ }^{(1)}$. Otra particularidad de este mercado laboral es la corta duración de los puestos de trabajo al vincularse con las temporadas de cosecha del producto agrícola ${ }^{(2)}$. Así, a lo largo del año, diferentes campañas que atraen a miles de personas en la búsqueda de un trabajo, desde la recolección del fruto rojo en Huelva hasta a la cosecha de la fruta dulce en Lleida durante el verano, pasando por la agricultura intensiva de mayor duración como la horticultura murciana y almeriense(3). El movimiento constante entre las diferentes explotaciones agrarias ha llevado a que este colectivo sea conocido como los temporeros.

Los trabajos de temporada se llevan a cabo principalmente en el campo para la recolección, el tratamiento y el desmochado de frutas y verduras; en estaciones agrícolas para la recolección; en explotaciones agrícolas o vitícolas; o en viveros. Las actividades del trabajo agrícola son numerosas: la cosecha, la poda, la recolección, el tratamiento, el acondicionamiento, etc. Por otro lado, también existe un importante grupo dedicado a las labores en naves o almacenes para el embalaje y almacenamiento del producto(2). El periodo de los contratos es variable según la región y la especie a recolectar. Mientras que en los campos del sur predominan las mujeres magrebíes, en los del norte lo hacen los hombres subsaharianos, y en los almacenes la mano de obra tiende a ser femenina y de Europa del Este ${ }^{(4)}$.

La temporalidad de estos trabajos conlleva la reducción de derechos. Aunque la legislación española reconoce diversos derechos a los trabajadores con nacionalidad extranjera, su situación de migrante supone, a menudo, una limitación de los mismos. Así, predomina una falta de protección legal, restricciones de acceso servicios sociales o sanitarios, poco respeto a la legalidad vigente, y condiciones laborales y habitacionales precarias. Un número significativo de migrantes trabaja sin afiliación a la Seguridad Social, y no tienen acceso a las prestaciones por desempleo(5).

La precariedad laboral del trabajo temporero se caracteriza por una elevada inestabilidad laboral, ausencia total de empoderamiento en tanto que no cuentan con un marco de protección legal, elevada vulnerabilidad agudizada por su situación legal y su estatus de inmigrante, nivel de ingresos insuficiente y más bajo que el de los trabajadores españoles, ausencia de derechos laborales y escaso poder para exigir mejores condiciones ${ }^{(6)}$. En este sentido, la fuerte competencia por los puestos de trabajo dificulta el asociacionismo y solidaridad entre el colectivo de trabajadores, incrementándose aún más su vulnerabilidad ${ }^{(7)}$.

La vulnerabilidad de los temporeros se materializa en las diversas situaciones de explotación que experimentan. Entre ellas destacan:

1) Los bajos salarios que perciben. Un estudio realizado en Lleida indicó que un $67 \%$ de quienes trabajaron en la campaña de la fruta en 2015 poseía un contrato en regla, con un salario en limpio de 5 euros la hora (74\%) -con una brecha de 
género de 1,73 € más para los varones (Figura 1)(2,8). A esto cabe sumar que no siempre se cobran todas las horas realizadas y que tampoco se trabaja a jornada completa durante todo un mes ${ }^{(8)}$. Además, los trabajadores migrantes de temporada suelen ser personas con cargas familiares, que dedican su salario a mantener, además de a sí mismos, hasta a cinco personas más (hijos, pareja, padres y/o otros familiares)(2). De hecho, de media los temporeros envían a sus familiares $291,69 €$ al mes, lo que reduce enormemente sus ingresos para su subsistencia durante su estancia en España ${ }^{(8)}$.

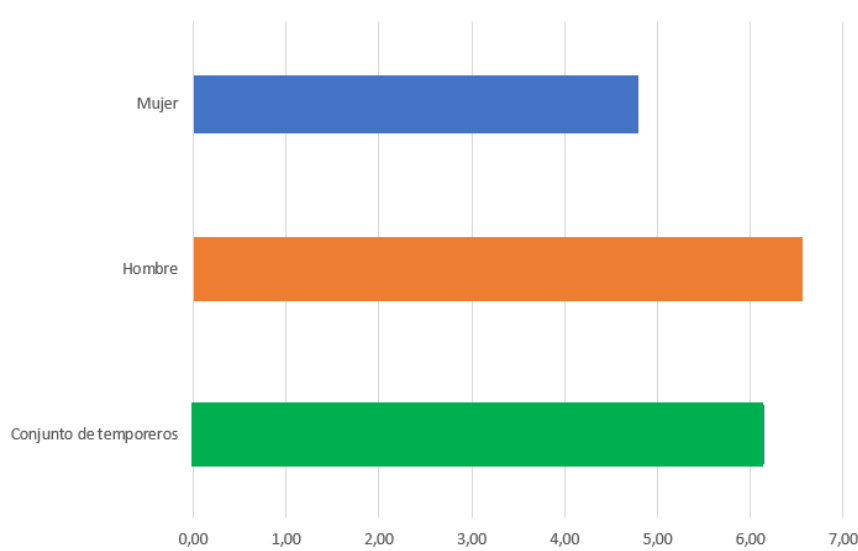

Figura 1. Salario medio por hora en limpio en el conjunto de temporeros en Lleida $(n=900)$ expresado en euros. Fuente: elaboración propia a partir de Garreta, et al (8).

2) Las horas de trabajo finalmente realizadas acostumbran a ser más que las que fueron pactadas originalmente con el empresariado, ya sea con o sin contrato. Por ejemplo, hasta 2,28 horas más por semana entre la jornada acordada con el empresario y las horas reales que finalmente se realizaron en los campos y almacenes de Lleida (Figura 2) ${ }^{(8)}$.

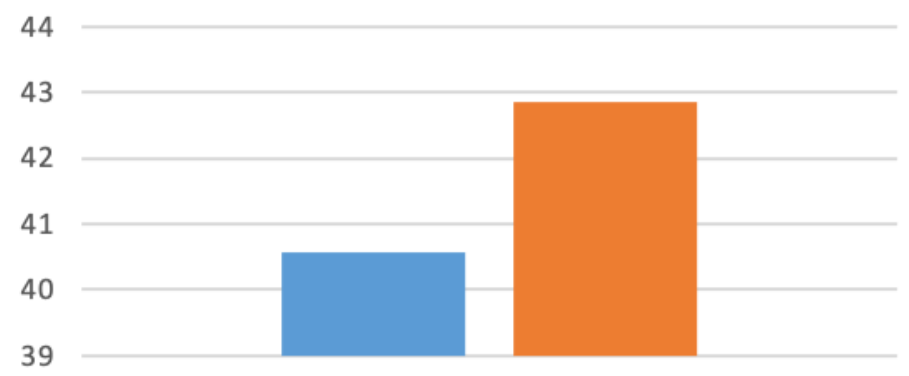

- Horas de trabajo acordadas $\quad$ Horas de trabajo realizadas

Figura 2. Horas de trabajo acordadas y horas de trabajo reales realizadas en una semana ( $n=900)$. Elaboración propia a partir del estudio de Garreta, et al.(8). 
3) Salvo para los contratos en origen, los empleadores no garantizan alojamiento, comida, ni el transporte hasta el lugar de trabajo. Según el convenio o acuerdo colectivo o el contrato, se puede deducir del salario la manutención y el alojamiento ${ }^{(9)}$. Muchos trabajadores permanecen en viviendas superpobladas y en zonas donde es difícil el acceso a la sanidad y los servicios básicos.

La situación de extrema precariedad que se genera en torno al trabajo agrícola se materializa en los asentamientos, un recurso habitual para los migrantes recién llegados a un territorio ${ }^{(10)}$. Los asentamientos, normalmente ubicados a las afueras de los entornos de actividad económica agrícola, son una estrategia de las personas migrantes para tener una vivienda temporal y que, a su vez, no les suponga un gasto económico que no tienen la seguridad de poder asumir. Aunque un $14 \%$ de los temporeros en Lleida reportaron vivir en infravivienda (como almacenes o asentamientos) $)^{(2,8)}$ en una realidad extendida en regiones de Andalucía, como Almería y Huelva.

Estos espacios habitacionales cuentan con una enorme cantidad de carencias. A nivel material, la falta de acondicionamiento a los fenómenos meteorológicos, la carencia de un mínimo de mobiliario e, incluso, el hecho de no tener acceso a servicios básicos como electricidad o agua corriente son una situación habitual en estos entornos. Esta realidad comporta un impacto en las condiciones de vida, la salud física y mental de las personas que en ellos habitan ${ }^{(10)}$.

\section{Impacto en la salud}

Estudios internacionales muestran que las condiciones de vida y trabajo precarias de las personas migrantes que trabajan en agricultura tienen consecuencias directas en su salud y calidad de vida ${ }^{(11-13)}$. Por una parte, el acceso a una vivienda digna y otros recursos básicos como la alimentación, dependen de la percepción de ingresos. Por otra, la salud autopercibida de los trabajadores agrícolas migrantes suele ser "pobre" o "baja" en comparación con la población general ${ }^{(14-15)}$. Están expuestos a un alto esfuerzo físico durante la jornada laboral, un tiempo de trabajo extenso y a un ritmo acelerado. Entre los problemas de salud más reportados en la literatura destacan los trastornos del sistema musculoesquelético, problemas dermatológicos como la dermatitis y el pruritoy las infecciones. ${ }^{(16-17)}$

Asimismo, estos trabajadores son susceptibles de sufrir lesiones y accidentes laborales. Sin embargo, no los denuncian por miedo a perder el empleo o a las represalias de los empresarios ${ }^{(15,16)}$. Estudios realizados en España e Italia muestran que las lesiones más frecuentes fueron las caídas desde una altura, seguidas de las lesiones con instrumentos cortantes/perforantes, las picaduras de insectos y serpientes, las caídas desde el mismo nivel y las lesiones con maquinaria ${ }^{(16,18)}$.

Además de la carga física, la carga psíquica derivada del trabajo y de las condiciones de vida fuera del trabajo, tienen un impacto negativo sobre la salud mental de las personas migrantes que trabajan en agricultura. Los síntomas físicos como el dolor lumbar y las cefaleas, la inseguridad laboral, la dificultad para acceder a los 
servicios sanitarios, las barreras lingüísticas, los conflictos con la población local, la intolerancia, la discriminación, las actitudes racistas, la falta de respeto y el aislamiento, aumentan la probabilidad de sufrir depresión, ansiedad y estrés ${ }^{(16)}$. Por ejemplo, en los invernaderos de Almería se ha detectado un alto riesgo de acoso laboral, sobre todo entre los trabajadores migrantes provenientes de América Latina y África; mientras que trabajadores de Europa del Este reportaron una falta de reconocimiento y una baja autonomía en sus trabajos ${ }^{(19)}$.

En el caso de las mujeres, éstas afrontan vulnerabilidades añadidas a las mencionadas anteriormente. Las mujeres que viven solas en asentamientos o cortijos están expuestas a sufrir diferentes tipos de violencia y abusos de parte de sus empleadores o compañeros, debido a las desigualdades de poder existentes, la prostitución o incluso la trata de seres humanos con fines de explotación sexual. Almería y Huelva destacan por la explotación sexual a la que son sometidas mujeres, conocida como "prostitución de invernadero o de cortijo"(20).

Por último, las personas migrantes, especialmente aquellas en situación administrativa irregular, se enfrentan a barreras de acceso a los servicios sanitarios $(6,11-12,21)$ que han sido agravadas durante la actual crisis sanitaria derivada de la pandemia del Covid19. Se sabe que, durante las crisis económicas, el deterioro de las condiciones de trabajo y empleo en los sectores más inestables como la agricultura, coloca a los trabajadores migrantes no calificados en una situación de gran inestabilidad produciendo efectos negativos en su salud ${ }^{(22)}$.

En conclusión, las personas migrantes temporeras ven afectada su salud debido a las malas condiciones laborales, habitacionales y de acceso a los recursos, junto a la desprotección social y jurídica. Urgen respuestas institucionales que garanticen una vida digna para estos trabajadores esenciales.

\section{Referencias}

1. Allepuz Capdevila R, Torres Solé T. La contratación de temporeros en las campañas de recolección del sector frutícola. Cuad rela labor. 2018;36(1):107-123. doi:10.5209/CRLA.59559

2. González JA, Garreta J, Llevot N. Trabajadores temporeros inmigrantes en el campo de Lleida (España): perfiles y situaciones sociolaborales. AGER. 2021;31:742. doi:10.4422/ager.2021.02

3. Gadea ME, de Castro C, Pedreño A, Moraes N. Jornaleros inmigrantes en la agricultura murciana: reflexiones sobre crisis, inmigración y empleo agrícola. Migraciones. 2015;(37):149-169. doi:10.14422/mig.i37.y2015.007

4. Gadea E, Pedreño A, Castro CD, Ramírez AJ. Almaceneras. Género y trabajo en los almacenes de manipulado de fruta en la región de Murcia. Revista Andaluza de Antropología. 2016;(11):121-145. 
5. González-Ortega S. La protección social de los trabajadores extranjeros. Expediente FIPROS-2006/94. [citado 30 Nov 2021]. Disponible en: https://www.seg-social.es

6. Porthé V, Benavides FG, Vázquez ML, Ruiz-Frutos C, García AM, Ahonen E, et al. La precariedad laboral en inmigrantes en situación irregular en España y su relación con la salud. Gac Sanit. 2009;23 Suppl 1:107-114.

7. Garreta i Bochaca J, Llevot Calvet N. El asociacionismo africano en España: una realidad incipiente. Migration studies. 2015;(197):141-157.

8. González, JA. Los temporeros de origen extranjero en las comarcas de Ponent: mercado de trabajo y migración. Lleida: Universitat de Lleida; 2018.

9. Recolección de fruta y Campaña de la vendimia 2021. Ministerio de inclusión, seguridad social y migraciones. Consejería de trabajo, migraciones y seguridad social en Francia [citado 1 Dic 2021]. Disponible en: https://www.mites.gob.es/

10. Asentamientos de inmigrantes. Una propuesta de intervención. CEPAIM, 2014. Disponible en: https://cepaim.org/documentos/publi/2-informe-final-Asentamientos-de-inmigrantes-propuesta-metodologica.pdf

11. Benach J, Vives A, Amable M, Vanroelen C, Tarafa G, Muntaner C. Precarious employment: understanding an emerging social determinant of health. Annual Review of Public Health. 2014;35:229-253. doi:10.1146/annurev-publhealth-032013-182500

12. Molina-Guzmán LP, Ríos-Osorio LA, Molina-Guzmán LP, Ríos-Osorio LA. Occupational health and safety in agriculture. A systematic review. Rev Fac Med. 2020;68(4):625-38. doi:10.15446/revfacmed.v68n4.76519

13. Sterud T, Tynes T, Mehlum IS, Veiersted KB, Bergbom B, Airila A, et al. A systematic review of working conditions and occupational health among immigrants in Europe and Canada. BMC Public Health. 2018; 18:1-15.

14. Rouland B. La santé des migrants internationaux dans la province d'Almería: indicateur de différenciations et d'inégalités. L'Espace Polit Rev [Edición electrónica]. 2015 [citado 3 Dic 2021]; 26. Disponible en: http://journals.openedition.org/ espacepolitique/3526.

15. Rouland B, Hoyez AC. Risques sanitaires et sociaux chez les travailleurs agricoles immigrés dans la province d'Almeria (Andalousie, Espagne). Espace Popul Sociétés. 2011;1:111-123. doi:10.4000/eps.4372

16. de Diego-Cordero R, Romero-Saldaña M, Jigato-Calero A, Badanta B, Lucchetti G, Vega-Escaño J. "Looking for Better (Job) Opportunities": A Qualitative Analysis of the Occupational Health of Immigrants in Southern Spain. Workplace Health Saf. 2021;69(5):198-207. doi: 10.1177/2165079920988005

17. Mucci N, Traversini V, Giorgi G, Garzaro G, Fiz-Perez J, Campagna M, et al. Migrant Workers and Physical Health: An Umbrella Review. Sustainability. 2019;11(1):232. doi: 10.3390/su11010232 
18. Cediel N, Conte V, Tomassone L, Tiberti D, Guiso P, Romero J, et al. Risk perception about zoonoses in immigrants and Italian workers in Northwestern Italy. Rev Saúde Pública. 2012;46(5):858-865. doi: 10.1590/S0034-89102012000500012

19. Montoya-García ME, Callejón-Ferre AJ, Pérez-Alonso J, Sánchez-Hermosilla J. Assessment of psychosocial risks faced by workers in Almería-type greenhouses, using the Mini Psychosocial Factor method. Appl Ergon. 2013;44(2):303-311. doi: 10.1016/j.apergo.2012.08.005.

20. Los derechos de las mujeres migrantes en Almería: la historia que nadie cuenta. Womens link worldwide [citado 4 Dic 2021]. Disponible en: https://www.womenslinkworldwide.org/files/1348/almeria-la-historia-que-nadie-cuenta.pdf

21. Agudelo-Suárez A, Gil-González D, Ronda-Pérez E, Porthé V, Paramio-Pérez G, García AM, Garí A. Discrimination, work and health in immigrant populations in Spain. Soc Sci Med. 2009;68(10):1866-1874. doi: 10.1016/j.socscimed.2009.02.046.

22. Ronda E, Briones-Vozmediano E, Galon T, García AM, García-Benavides F. Agudelo AA. A qualitative exploration of the impact of the economic recession in Spain on working, living and health conditions: reflections based on immigrant workers' experiences. Health Expect. 2016 Apr;19(2):416-26. doi: 10.1111/hex.12365 